\title{
Bridging UMTS and Multicast Routing in ns-2
}

\author{
Antonios Alexiou \\ Research Academic Computer \\ Technology Institute and \\ Computer Engineering \& Informatics \\ Department, University of Patras \\ GR 26500, Patras, Greece \\ 0030-2610996954 \\ alexiua@cti.gr
}

\author{
Christos Bouras \\ Research Academic Computer \\ Technology Institute and \\ Computer Engineering \& Informatics \\ Department, University of Patras \\ GR 26500, Patras, Greece \\ 0030-2610996951
}

\author{
Andreas Papazois \\ Computer Engineering \& Informatics \\ Department, University of Patras \\ GR 26500, Patras, Greece \\ 0030-2610422494 \\ papazois@ceid.upatras.gr
}

\begin{abstract}
In this paper, we present the design and the implementation of a novel scheme for the multicast transmission of data over Universal Mobile Telecommunications System (UMTS) networks. The proposed scheme is implemented as an ns-2 network simulator module which can be employed to investigate various aspects of UMTS multicast. Apart from the normal multicast transmission over UMTS, we consider the handling of exceptional cases induced by user mobility scenarios. The proposed scheme is in accordance with the current specifications of the Multimedia Multicast/Broadcast Service (MBMS) defined by the 3rd Generation Partnership Project (3GPP) and introduces minor modifications in the UMTS architecture and the mobility management mechanisms. The performance of the proposed scheme is validated and analyzed through simulation experiments. Finally, the simulation results are presented along with their analysis.
\end{abstract}

\section{Categories and Subject Descriptors}

C.2.1 [Computer-Communication Networks]: Network Architecture and Design -packet-switching networks, wireless communication; I.6.4 [Simulation and Modeling]: Model Validation and Analysis

\section{General Terms}

Algorithms, Measurement, Performance, Design, Reliability, Experimentation, Verification.

\section{Keywords}

Modeling, Simulation, Multicast, UMTS, Mobility, MBMS.

\section{INTRODUCTION}

Multicast is an efficient method for data transmission to multiple destinations. Its advantage is that the sender's data are transmitted only once over the links which are shared along the paths to a

Permission to make digital or hard copies of all or part of this work for personal or classroom use is granted without fee provided that copies are not made or distributed for profit or commercial advantage and that copies bear this notice and the full citation on the first page. To copy otherwise, to republish, to post on servers or to redistribute to lists, requires prior specific permission and/or a fee. servers or to redistribute to lists, requires prior specific
SIMUTOOLS 2008, March 03-07, Marseille, France SIMUTOOLS 2008, March 03-07, Marseille, France Copyright @ 2008 ICST 978-963-9799-20-2 targeted set of destinations. Data duplication is restricted only in nodes where the paths diverge to different subnetworks [2]. 3GPP identified the need for multicast routing in UMTS networks and started the standardization of MBMS framework [1]. In the meantime, several researchers, like the authors of [2], [6] and [7], have investigated the requirements and proposed mechanisms for the multicast transmission over UMTS networks. However, the performance of the proposals was assessed through analytical models and processes. No simulation experiment has been conducted to validate the service provision along with the impact of user mobility over the service continuity

In this paper, we present the design and the implementation of a novel scheme for the multicast transmission of data over UMTS networks. Our proposed scheme is implemented as an ns-2 network simulator module. The innovation of our work stems from the introduction of a module that bridges the UMTS network simulation and the multicast data transmission in the widely used ns-2 network simulator. Our contribution is a platform that can be employed by researchers to validate and analyze multicast mechanisms over UMTS networks. Some areas of active research that may be boosted by the deployment of our module are the MBMS service congestion control, the UMTS multicast group management, the multicast radio resource management or the MBMS Quality of Service (QoS).

Apart from the normal multicast transmission of data over UMTS, we consider the handling of special cases induced by user mobility scenarios. User mobility is the distinctive feature of the cellular networks and it has been our belief and the motivation behind our study that user mobility situations are of major importance and must be thoroughly examined through simulation. Our proposed scheme can cope with the user mobility without any disruption of the service provision or any packet loss. Consequently, another important contribution of our work is a platform where user mobility scenarios may be validated and analyzed through simulation.

This paper is structured as follows: Section 2 presents an overview of the standardized MBMS service provision. In Section 3 , we analyze the design of the proposed scheme. Section 4 describes the implementation of this new scheme in ns-2. Section 5 is dedicated to the simulation results. Finally, some concluding remarks and planned next steps are briefly described in Section 6 . 


\section{OVERVIEW OF UMTS AND MBMS 2.1 Basic UMTS Architecture}

The basic UMTS network is split in two parts: the User Equipment (UE) and the Public Land Mobile Network (PLMN). The PLMN is further divided into two land-based infrastructures: the UMTS Terrestrial Radio-Access Network (UTRAN) and the Core Network (CN) (Figure 1). The UTRAN handles all the radio-related functionalities. The $\mathrm{CN}$ is responsible for maintaining subscriber data and for switching voice and data connections.

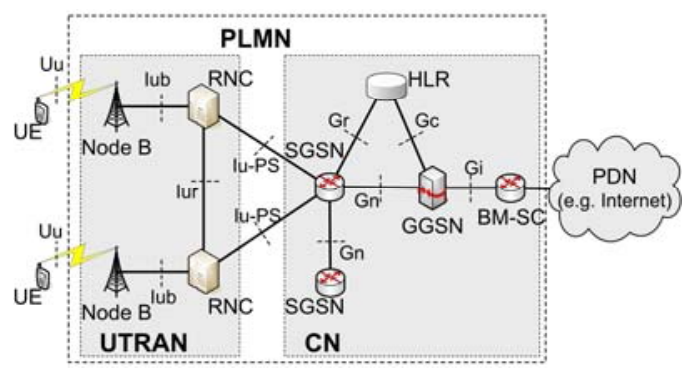

Figure 1. UMTS architecture for MBMS service.

The UTRAN consists of two kinds of nodes: the Radio Network Controller (RNC) and the Node B. The Node B constitutes the base station and provides radio coverage to one or more cells. A single RNC and the Node Bs which it controls constitute a Radio Network Subsystem (RNS). The CN is logically divided into the Circuit-Switched (CS) domain and the Packet-Switched (PS) domain. All of the voice related traffic is handled by the CSdomain, while the PS-domain handles the transfer of data packets. The PS-domain is more relevant to the multicast data transmission which is the scope of this paper and, therefore, in the remainder we shall focus on the PS-functionality. The PS-domain of the CN consists of two kinds of General Packet Radio Service (GPRS) Support Nodes (GSNs), namely the Gateway GSN (GGSN) and the Serving GSN (SGSN).

\subsection{MBMS Service}

As we mentioned above, the 3GPP has initiated the standardization of the MBMS service. The basic MBMS architecture is almost the same as the existing UMTS architecture in the PS-domain. The most significant modification is the addition of a new node called Broadcast Multicast-Service Center (BM-SC) (Figure 1). In this node the MBMS data are scheduled and interfaces are provided for the interaction with the content provider. In order to reduce the implementation costs, the intention of the 3GPP is to limit the changes introduced in the existing radio and core network architectures. For simplicity reasons, in our analysis we shall consider the functionality of the BM-SC incorporated in the GGSN.

There are two types of MBMS service mode: the broadcast and the multicast. In broadcast mode, data are delivered to a specified area without knowledge of the receivers and whether there is any receiver at all in this area. Since the multicast mode is more general than the broadcast one, we shall present the operation of the MBMS multicast mode and the way that the mobile user receives multicast data. The reception of an MBMS multicast service is enabled by certain procedures. These are: Subscription,
Service Announcement, Joining, Session Start, MBMS Notification, Data Transfer, Session Stop and Leaving.

\section{THE PROPOSED SCHEME}

In this section, we present the scheme for the multicast transmission of data over UMTS. Furthermore, we analyze the handling of special cases caused by user mobility scenarios.

\subsection{Packet Forwarding Mechanism}

If we consider the GGSN node as root then we may conclude that the network topology of the UMTS has a tree-like form. Our multicast mechanism takes advantage of the tree-like topology of the UMTS networks and introduces Routing Lists (RLs) in every node of the network apart from the UEs. In the RL of a node, information is kept about which nodes of the lower level connect the current node with the UEs belonging to a specific multicast group. Consequently, there is one RL for each multicast group in each node (except for the UEs). The packet forwarding during the Data Transfer phase is based on the RL processing in each node. If an incoming multicast packet, reaches a node, the corresponding RL is scanned. If RL is non-empty, the packet is duplicated and is transmitted once to each lower-level node existing in the RL. This procedure is repeated recursively in the lower-level nodes until each copy of the packet reaches its destination.

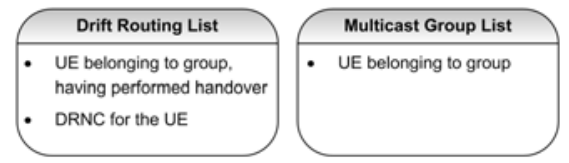

Figure 2. Additional types of lists.

In this point, we must mention the existence of two other kinds of lists. Additionally to the RLs, the Drift Routing Lists (DRLs) are used in the RNCs and the Multicast Group Lists (MGLs) in the GGSN (Figure 2). The DRLs are lists which are used when interRNS soft handover has occurred. Each DRL corresponds to a multicast group and contains pairs of RNC-UE. The multicast transmission over the Iur interface (see Figure 1) is achieved through the DRLs and will be thoroughly explained in Section 3.3. Each MGL corresponds to a specific multicast group and maintains the UEs belonging to the group. These lists enable GGSN to retrieve the UEs belonging to a specific multicast group. Obviously, MGLs and RLs are not static elements but they are updated each time a UE joins or leave a multicast group or when user mobility events transform the mobile network topology.

\subsection{Multicast Group Management}

Consider a UMTS network providing an MBMS service. Suppose that a UE has completed the Subscription phase and wants to join a multicast group provided. In this case, the Service Announcement phase is executed. UE sends a message to the GGSN, requesting the list of available multicast groups. When the message reaches the GGSN, the GGSN sends a reply message to the UE with the available multicast groups and the Service Announcement phase ends. The UE decides which multicast group(s) wants to join in. The Joining Phase consists of the following steps: 
1. The UE sends a join-request message to the GGSN, specifying the multicast group in which the UE wants to join in.

2. The GGSN checks the subscription profile. If we suppose that the GGSN accepts the request, the UE is added in the corresponding MGL. Then, it checks if the SGSN which serves the UE, exists in the relevant RL. If it does not exist, it adds it in the RL. Finally, the GGSN sends an acknowledgement message to the serving SGSN.

3. When the SGSN gets the acknowledgment, it examines if the serving RNC of the UE exists in the RL that it maintains for the mg. If the RNC does not exist, the SGSN adds it in the RL. Then it forwards the acknowledgment to the RNC.

4. When the RNC gets the acknowledgment, it adds the UE in the proper RL and forwards the acknowledgment to the UE.

When a UE decides to leave a multicast group, the Leaving phase takes place. The message sequence during this phase is similar to the one described above.

\subsection{User Mobility Handling}

Consider a UE that is a member of a multicast group and a MBMS multicast service provision that is in the Data Transfer phase. While the multicast packets are being transmitted to the members of this multicast group, the specific UE moves in another cell. Supposing that this cell is controlled by a different RNC, an inter-RNS soft handover will be performed. In this case, the UE receives data simultaneously from the two involved Node Bs. The basic concept of inter-RNS soft handover is that the handover is transparent to the SGSN(s). Thus, when inter-RNS handover takes place the data is transmitted through the Iur interface (Figure 1). The proposed mechanism introduces the DRLs for the multicast transmission over the Iur interface. Multicast over the Iur interface takes place when multiple handovers from the same source RNS to the same target RNS have occurred.

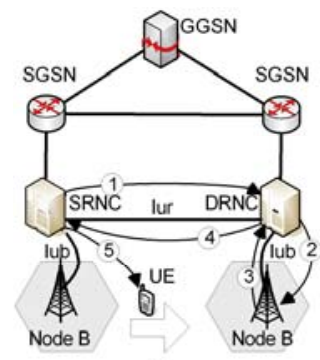

(a)

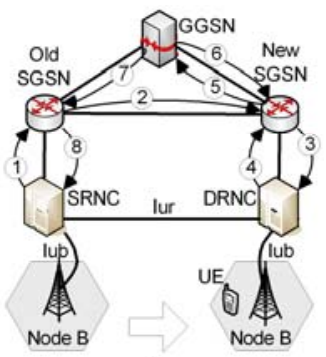

(b)
Figure 3. User mobility handling: (a) inter-RNS handover and (b) inter-SRNS relocation.

The source RNC is called Serving RNC (SRNC) and the target RNC is called Drift RNC (DRNC). Figure 3(a) describes the steps of the inter-RNS soft handover procedure. The proposed mechanism is based on the existing handover procedure of UMTS but it incorporates several extensions in order to assure MBMS service continuity. These extensions are pointed out during our analysis.
1. The SRNC decides to make a handover based on the measurements from the UEs. An Iur connection is established between SRNC and DRNC and the SRNS requests radio link.

2. If radio resources are available, the DRNC forwards the request to the Node $B$ in which the new cell belongs.

3. When the allocation of the radio resources is completed, the Node B sends an acknowledgement message to the DRNC and starts receiving uplink data from the UE. When the DRNC gets the acknowledgement, it adds the UE in the RLs related to the multicast groups that the UE belongs. Then, the DRNC forwards the acknowledgment to the SRNC.

4. When the SRNC gets the acknowledgment, it inserts the UE/DRNC pair in the DRLs related with the multicast groups that the UE belongs in

5. Finally, the UE is informed of the handover and receives the connection information. Now the UE receives the same data from the two involved Node Bs.

Regarding the packet forwarding mechanism, an additional check must be made in the RNCs. The new functionality is related to the existence of the Iur connections and it is based on the DRLs processing. In more detail, if a multicast packet reaches an RNC, not only the corresponding RL but also the corresponding DRL is scanned. If DRL is non-empty, the packet is duplicated and is transmitted once to each DRNC existing in the DRL. These transmissions are made over the corresponding Iur interfaces and follow the multicast forwarding concept. If a UE has performed subsequent handovers, multiple DRNCs correspond to its connection. In that case, this procedure is repeated recursively in the DRNCs until of the packet reaches the last DRNC. Finally, this DRNC will transmit the packet to the UE.

Consider the previous scenario and suppose that, after a time interval, the UE is outside the coverage area of its first cell. In this case, a SRNS relocation procedure is triggered. This procedure is used to move the UTRAN-to-CN connection point from the SRNC to the DRNC. If the DRNC is connected to the same SGSN as the SRNC, an intra-SGSN SRNS relocation procedure is performed. Otherwise an inter-SGSN SRNS relocation procedure takes place. Figure 3(b) illustrates the latter case which is the most general of the two. Note that the proposed mechanism uses existing mobility management mechanisms but with several extensions for multicasting.

1. The current SRNC detects that SRNS relocation of the UE to a DRNC is needed. The SRNC sends a "relocation required" message to the old SGSN which indicates that the SRNC is the source RNC and the DRNC is the target RNC.

2. The old SGSN sends message "relocation request" to the new SGSN. Additionally, the new SGSN gets aware of the multicast groups in which the UE participates. The MBMS related context is also transferred.

3. The new SGSN sends message "relocation request" to the target RNC. The target RNC is informed by the new SGSN in which multicast groups the UE belongs. Finally, the target RNC performs an update of the relevant RLs, although this is not mandatory because the UE has already been inserted in the RLs during the soft handover procedure 
4. A "relocation-ack" message is sent from the target RNC to the new SGSN. The new SGSN examines each RL related with the multicast groups that the UE participates. If the target RNC is not contained, it is added in the RL.

5. The new SGSN creates MBMS bearer context and registers on the GGSN. The GGSN examines each RL related with the multicast groups that the UE participates. If the new SGSN is not contained, it is added in the RL.

6. GGSN notifies the new SGSN of the UE registration. The connection is now switched from old SGSN to new SGSN.

7. The GGSN notifies the old SGSN that the service is deregistered for the specific UE. The old SGSN examines each $\mathrm{RL}$ related with the multicast group that UE participates. If there is no other UE which is served by the source RNC and participates in the corresponding multicast group, source RNC is erased from the RL.

8. The source RNC is notified by old SGSN that relocation is completed. All the records containing the UE are removed from all the DRLs and RLs of source RNC. From now on the target RNC is considered to be the SRNC of the UE.

The case of intra-SGSN SRNS relocation is similar. All the above steps are valid given that both SRNC and DRNC are connected to the same SGSN.

\section{THE nS-2 MODULE DESCRIPTION}

As we mentioned above, the proposed scheme was implemented as an ns-2 network simulator module. In this section we describe some implementation aspects along with a short description of the way our module can be deployed and used.

\subsection{The ns-2 Network Simulator}

Ns-2 is an open-source tool which is very commonly used by the international scientific community. It constitutes a very powerful simulation environment and is able to simulate a very wide range of networks, including wireless networks. The simulator is written in $\mathrm{C}++$ programming language. Additionally, OTcl scripting language is used as a command and configuration interface. Ns-2 supports all the basic network protocols (like TCP, UDP and RTP), several types of traffic (like FTP, Telnet, HTTP, CBR and VBR), queue management schemes (like RED, DropTail and $\mathrm{CBQ}$ ) and routing algorithms (like Dijkstra and Bellman Ford [4].

Ns-2 does not natively support the UMTS network functionality. Therefore, it was patched with its EURANE extension. The Enhanced UMTS Radio Access Network Extensions for ns-2 (EURANE) is a set of extensions which implement the three UTRAN nodes which ns-2 does not support. In more detail, the EURANE enables the declaration of RNC, Node B and UE nodes and simulates realistically their functions. Moreover, this extension enables the declaration of the following UTRAN transport channels: FACH, RACH, DCH and HS-DSCH [3].

\subsection{New Implementation}

Multicast routing is natively supported in ns-2 for many protocols and node types. Additionally, the EURANE extension for ns-2 offers an almost complete support of unicast transmission over UMTS. Although the MBMS service standardization has already started, no extension for ns-2 has been implemented for the support of multicast routing in UMTS. Consequently a major motivation of our work was the implementation of a module that bridges multicast data transmission and UMTS networks simulation. This module can boost the research at the specific domain and the full specification of the MBMS service.

Our introduced module implements in $\mathrm{C}++$ programming language the multicast packet forwarding scheme described in the previous section. In order to implement this module, first of all, we had to introduce the RLs in each node of the UMTS network except for the UEs. Thus, we created a new class named Routing_List. Moreover, we used inheritance in order to define two new sub-classes derived from super-class Routing_List. These subclasses are named Multicast_Group_List and Drift_Routing_List and represent the MGLs in the GGSNs and DRLs in the RNCs respectively.

Every list is a double linked list which contains the appropriate methods in order to be read and modified. In an SGSN, for example, we are able to retrieve the nodes of the next level (RNCs) that are served by the same SGSN and are members of the multicast group $m \_$group_id by calling the Routing_List class method Get_Next_Level_Nodes(m group_id). In the GGSN, a method of the sub-class Multicast_Group_List called Get_UEs(m_group_id) is provided returning the UEs that are members of the multicast group indicated by the argument m_group_id. Similarly, the sub-class Drift_Routing_List owns a method called Get_Drift_RNCs(m_group_id).

Likewise, there are methods that update appropriately the lists. For instance, in every node there is a method called Set_Next_Level_Node(m_group_id, next_level_node, UE_id), that adds the next level node to the Routing_List that is related with the argument $m \_$group_id. Obviously, in case that the related Routing_List has not been created yet, the above method creates a new Routing_List related with the $m \_$group_id and then inserts the item in it. For the case of the removal of a next level node, the method Remove_Next_Level_Node(m_group_id, next_level_node, $\left.U E \_i d\right)$ is used. Similarly, there are relevant methods which update the MGLs and DRLs.

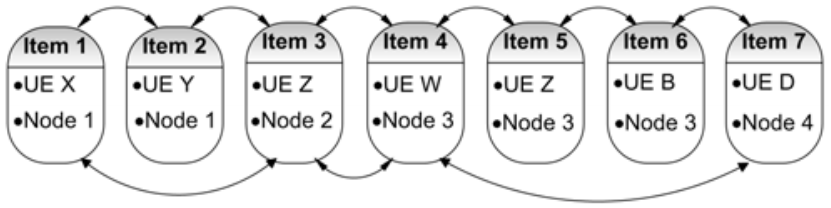

Figure 4. Two-way linkage of the items in DRLs.

Due to the association of each item of the Routing_List with a specific UE through the attribute UE_id, the execution of the method Get_Next_Level_Nodes(m_group_id) would be rather ineffective. For the fast access and modification of the Routing_List, the items with different next_level_node attribute are linked with a second level of links (this level of links is appeared below the list items in Figure 4). This implementation increases efficiency when the current node receives traffic. There is no need to scan the whole file; only the second level links are examined. The same approach has been followed in order to increase efficiency during the scanning of Drift_Routing_List. 
The next step of the implementation was to create the mechanism which fills these lists and to organize the procedures described in Section 3. The method Set_Next_Level_Node(m_group_id, next_level_node, $\left.U E \_i d\right)$ is executed when a Joining phase takes place. Instead, when a Leaving phase takes place the Remove_Next_Level_Node(m_group_id, next_level_node, UE_id) is employed to remove the relevant item. For the communication of the nodes, as well as the transmission of the multimedia data we used RTP/RTCP messages. Since the RTP/RTCP protocols are fully functional in the EURANE module, only minor modifications were introduced to the RTP/RTCP protocol implementation. The purpose of these modifications was to include some additional information in the RTP/RTCP packet headers. In more detail, this information includes the type of the request/response, the $m \_g r o u p \_i d$, the $U E \_i d$ as well as the Quality of Service (QoS) profile of the associated UEs.

\section{SIMULATION EXPERIMENTS}

\subsection{Simulation Topology and Parameters}

For our simulation model, we considered the topology presented in Figure 5. We assumed that the external node connected to the GGSN, was a media server which imported the multicast video traffic in our examined UMTS network. This traffic was addressing to a specific multicast group of UEs. The number of UEs which consisted the multicast group during the experiments, varied from 20 to 200. Nevertheless, the performance of our proposed scheme was independent of the multicast group size. Since we did not want to create a symmetrical topology, we differentiated the number of the UEs located in each Node B service area. For the sake of simplicity, we considered that there was only one multicast group which the UEs of the network could join. The video data was transmitted in the form of RTP traffic. This means that, with the use of the RTCP reports, the GGSN had the opportunity to gain useful statistical information. The bit rate of the video was 256 kbps and the packet size was 512 bytes.

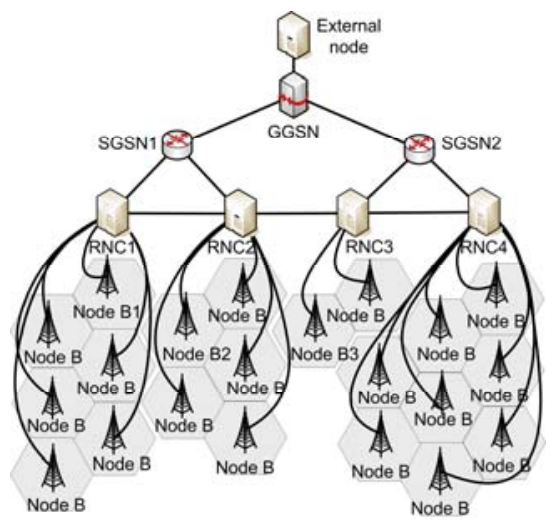

Figure 5. Simulation topology.

\subsection{Simulation Results}

In the first place, we calculated the bandwidth consumption in the links of the UMTS network when the multicast scheme is used for the video transmission. Then, we compared it with the bandwidth consumption in the same link when multiple unicast transmission is used. In Figure 6, we present the result over the links GGSNSGSN1 and SGSN1-RNC1. In the first case we supposed that the recipients are 10 UEs which have joined the multicast group and are served from SGSN1. In the second case, the recipients are 5 UEs which have joined the multicast group and are located in the RNS controlled by RNC1.

As we can observe from the Figure 6, during the multicast transmission, the bandwidth consumption in these links was approximately equal to the theoretical video bit rate (256kbps) represented by the straight line. This occurs because in the multicast scheme, the packets are transmitted only once over each link of the network until they reach the mobile users. Instead, in the unicast scheme the same packets are transmitted multiple times over every link. This means that the bandwidth consumption increases. In more detail, we conclude that the fraction of the difference of the two lines, depicted in the figures, depends on the number of the members of the multicast group. This bandwidth consumption gain is the main property and the most important benefit of the multicast data transmission.

The other aspect that we examined was whether the service continuity is secured by our scheme. By the term service continuity, we mean that all packets are delivered correctly to all receivers without any disruptions of the service. Apart from the normal case when multicast data transmission is taking place, we analyzed the most critical phase for service disruption which is during user mobility scenarios.

In order to examine the service continuity during user mobility scenarios, we simulated the following case. We considered the previously described video traffic of 256kbps and a UE located in the cell served by Node B1 (Figure 5). After 20 seconds of receiving multicast data, the examined UE performed a handover to the cell served by Node B2. Obviously, an inter-RNS/intraSGSN soft handover took place. The packets were transmitted to RNC2 through the Iur interface which connects RNC1 and RNC2. At 40 seconds, SRNS relocation was requested from RNC1 and, when the procedure had been completed, the RNC2 was the new SRNC of the UE and the Iur was released for that session. At 60 seconds a new handover took place. That time it was an interRNS/inter-SGSN soft handover to the cell served by Node B7. Finally, a second SRNS relocation was requested. This request came from RNC2 and the target RNC was RNC3. During the relocation procedure the Iur interface between RNC2 and RNC3 was released and RNC3 was the SRNC of the UE. The simulation stopped at 100 seconds. During this time, we calculated the bit rate of the data received by UE1.

We can observe that the bit rate of the data received from the UE is stable during the above described scenario. Moreover, analysis of the simulation log shows that no packet loss took place during the whole procedure. Instead, all the packets addressing to the multicast group were properly delivered to the moving UE. Thus, we can conclude that the service continuity is secured. 


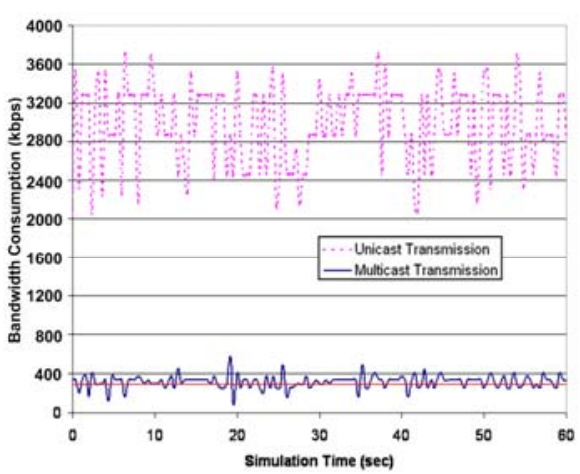

(a)

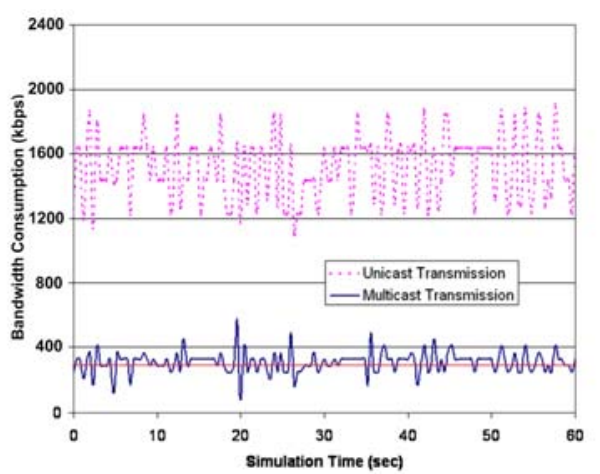

(b)

Figure 6. Bandwidth consumption in: (a) GGSN-SGSN1 and (b) SGSN1-RNC1 links.

Finally, we examined the multicast transmission of video over the Iur interface. We supposed that multiple handovers had taken place from the same source RNC to the same target RNC. We considered the same network topology of Figure 5 and we supposed that 4 UEs were located in the RNS controlled by RNC1. These UEs are members of a multicast group and MBMS service provision for that group is in the Data Transfer phase. During this simulation 4 subsequent inter-RNS handovers took place at 20,40, 60 and 80 seconds respectively. We supposed that the new cells are located in the RNS controlled by RNC2 and that the handovers were not followed by relocations. The simulation was terminated at 100 seconds. In this case, we calculated the bandwidth consumption in the Iur interface connecting the RNC1 with RNC2. Then, we compared the results with the bandwidth consumption in the same link when multiple unicast is used. Figure 7 visualizes the result of the comparison.

Figure 7 depicts clearly the efficiency in the use of the Iur interface of our proposed multicast scheme. It is obvious that the bandwidth consumption remains stable without depending on the number of handovers. On the contrary, the existing multiple unicast mechanism causes increment of the transmitted load, making this interface a potential bottleneck.

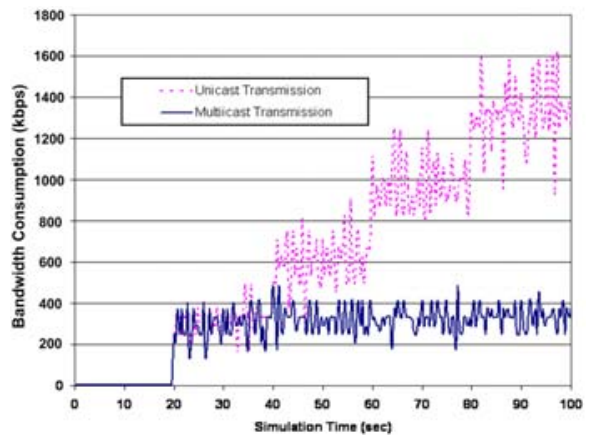

Figure 7. Multicast transmission over Iur interface.

\section{CONCLUSIONS AND FUTURE WORK}

In this paper, we proposed a novel scheme for the multicast delivery of data over UMTS networks. Our scheme is based on the existing UMTS infrastructure and, therefore, minor modifications are needed in the UMTS architecture and the mobility management mechanisms. The proposed scheme was implemented as an ns-2 network simulator module which bridges multicast routing with UMTS network simulation. We described the implementation of our module along with its use. The module can be easily installed and configured as an ns-2 extension. Additionally, through simulation experiments we validated and analyzed our module under various network topologies and user distributions. The result of our work is a platform that can support multicast routing over UMTS along with user mobility scenarios. This platform could constitute the basis for research and a large scale of experiments related to this scientific domain. A step that follows this work could be the study of congestion control schemes for the multicast data transmission over UMTS networks.

\section{REFERENCES}

[1] 3GPP. 2007. Multimedia Broadcast/Multicast Service (MBMS); Architecture and functional description (Release 7), Technical Specification 23.246 v7.2.0.

[2] Alexiou, A., Antonellis, D., Bouras, C., and Papazois, A. 2006. An Efficient Multicast Packet Delivery Scheme for UMTS. In Proceedings of the 9th ACM International Symposium on Modeling Analysis and Simulation of Wireless and Mobile Systems (Malaga, Spain, October 2-6, 2006). MSWiM'06. ACM Press, New York, USA, 147-150.

[3] Ericsson Telecommunicatie B.V. 2005. User Manual for EURANE. The SEACORN Project.

[4] Fall, K., and Varadhan K. 2007. The ns Manual. The VINT Project.

[5] Holma, H., and Toskala, A. 2004. WCDMA for UMTS: Radio Access for Third Generation Mobile Communications (3rd Edition), John Wiley and Sons Ltd.

[6] Ivancovic, T. 2005. Support of Multimedia Broadcast/ Multicast Service in UMTS Networks. In Proceedings of the 8th International Conference on Telecommunications (Zagreb, Croatia, June 15-17, 2005) ConTEL’05. University of Zagreb, 91-98.

[7] Rummler, R., Chung, Y., and Aghvami, H. 2005. Modeling and Analysis of an Efficient Multicast Mechanism for UMTS. IEEE Transactions on Vehicular Technology. 54, 1 (Jan. 2005), 350-365. 